\title{
Pressure Dependence of Diffusion in Simple Glasses and Supercooled Liquids
}

\author{
H. R. Schober \\ Institut für Festkörperforschung, Forschungszentrum Jülich, D-52425, Germany
}

(Received 12 November 2001; published 25 March 2002)

\begin{abstract}
Using molecular dynamics simulation, we have calculated the pressure dependence of the diffusion coefficient in a binary Lennard-Jones glass. We observe four temperature regimes. The apparent activation volume drops from high values in the hot liquid to a plateau value. Near the critical temperature of the mode coupling theory it rises steeply, but in the glassy state we find again small values, similar to the ones in the liquid. The peak of the activation volume at the critical temperature is in agreement with the prediction of mode coupling theory.
\end{abstract}

DOI: 10.1103/PhysRevLett.88.145901

Diffusion in glasses and their melts has been studied intensively for many years. These efforts are stimulated both by the technological importance of glassy and amorphous materials and by the desire to understand the physics of disordered systems in general and the liquid to glass transition in particular. Despite this effort there is still no agreement on the nature of diffusion on an atomic level or on its change at temperatures near the glass transition. This even holds for simple densely packed glasses, such as binary metallic ones; see for recent reviews Refs. [1-5].

In a hot liquid, diffusion is by flow, whereas, in the glass well below the transition temperature, it will be mediated by hopping processes. One key question is the transition between the two regimes. For fragile glasses, such as most polymers and amorphous metallic glasses, mode coupling theory (MCT) predicts an arrest of the homogeneous viscous flow in the undercooled melt at a temperature $T_{c}$, well above the glass transition temperature $T_{g}$ [6]. Hopping processes will suppress the predicted singularities and will become the dominant diffusion process near $T_{c}$.

The nature of the hopping process is another issue of controversy. Is it by a vacancy mechanism, similar to diffusion in the crystalline state, or is it via a collective process inherent to the disordered structure? Investigations are hampered by the fact that glasses are thermodynamically not in equilibrium, and one observes aging. The diffusion coefficient of a glass which has been relaxed for a long time will be considerably lower than the one of an "as quenched" glass.

In crystalline materials the pressure dependence of the diffusion coefficient can often be used to identify the diffusion mechanism. For thermally activated diffusion the diffusion coefficient can be described by an Arrhenius law

$$
D(T)=D_{0} \exp (-H / k T),
$$

where $D_{0}$ is a preexponential factor and $H$ is the activation enthalpy. In a more component system the diffusion coefficients and the parameters describing them will be different for each component. For simplicity's sake we drop the indices indicating the component here and in the following, whenever possible.
PACS numbers: 66.10.-x, 61.43.Fs, 64.70.Pf, 66.30.-h

Using $V=\partial G / \partial p$ with $G=H-T S$ one obtains the activation volume for a diffusion by a single jump process [7]

$$
V_{\mathrm{act}}=-k T\left[\frac{\partial \ln D}{\partial p}\right]_{T}+k T\left[\frac{\partial \ln D_{0}}{\partial p}\right]_{T} .
$$

In crystals the second term is only a minor correction which can be neglected. For diffusion mediated by defects, the activation volume splits into two terms, $V_{\text {act }}=$ $V^{f}+V^{m}$, a formation volume $V^{f}$, and a migration volume $V^{m}$ For diffusion via thermal vacancies the formation volume dominates and $V_{\text {act }}$ varies between $0.6 \Omega$ and $1 \Omega$, where $\Omega$ is the average atomic volume. For the migration part one estimates $V^{m} \sim 0.1 \Omega$. As a result, in crystals high values of $V_{\text {act }}$ are taken as a signature of a thermally activated diffusion mediated by thermal vacancies.

Assuming that also in the glass the first term in Eq. (2) dominates, one usually describes, also in amorphous materials, the pressure dependence of diffusion by an apparent activation volume

$$
\hat{V}_{\mathrm{act}}=-k T\left[\frac{\partial \ln D}{\partial p}\right]_{T} .
$$

Experiments on a number of metallic glasses give a large spread of values in the range of $(0.05-1) \Omega$ [5]. Low values were, e.g., observed in $\mathrm{Co}_{81} \mathrm{Zr}_{19}$ [8] where no significant isotope effect is observed [9]. This result can be interpreted in terms of a collective diffusion mechanism which does not involve "defects" as diffusion vehicles and is inherent to the glassy structure. The situation is not so clear for the case of large activation volumes $\hat{V}_{\text {act }}$. The activation volume of $0.9 \Omega$ for diffusion of $\mathrm{Zr}$ in $\mathrm{Co}_{9} 2 \mathrm{Zr}_{8}$ was interpreted as an indication of diffusion via vacancy-like defects [8] rather than by an inherent mechanism as in the case of Co. Values of around $0.5 \Omega$ have been observed in several materials [10-12]. Such values are also found in materials where the vanishing isotope effect points to diffusion by collective jumps [13]. Whether collective diffusion not involving "diffusion vehicles" can give rise to activation volumes of the order $(0.05-1) \Omega$ is still open. 
In the liquid state the diffusion coefficient can be fitted by a Vogel-Fulcher-Tammann law (VFT)

$$
D^{\mathrm{VFT}}(T)=D_{0}^{\mathrm{VFT}} \exp \left[-E^{\mathrm{VFT}} / k\left(T-T^{\mathrm{VFT}}\right)\right] .
$$

From this we get the activation volume

$$
\begin{aligned}
\hat{V}_{\mathrm{act}}^{\mathrm{VFT}}=-k T[ & \frac{\partial \ln D_{0}^{\mathrm{VFT}}}{\partial p}-\frac{1}{k\left(T-T^{\mathrm{VFT}}\right)} \frac{\partial E^{\mathrm{VFT}}}{\partial p} \\
& \left.-\frac{E^{\mathrm{VFT}}}{k^{2}\left(T-T^{\mathrm{VFT}}\right)^{2}} \frac{\partial T^{\mathrm{VFT}}}{\partial p}\right]_{T} .
\end{aligned}
$$

From MCT one derives [6] alternative expressions

$$
\begin{gathered}
D^{\mathrm{MCT}}(T)=D_{0}^{\mathrm{MCT}}\left(T-T_{c}^{\mathrm{MCT}}\right)^{\gamma}, \\
\hat{V}_{\mathrm{act}}^{\mathrm{MCT}}=-k T\left[\frac{\partial \ln D_{0}^{\mathrm{MCT}}}{\partial p}-\gamma \frac{1}{\left(T-T_{c}^{\mathrm{MCT}}\right)} \frac{\partial T_{c}^{\mathrm{MCT}}}{\partial p}\right. \\
\left.+\ln \left(T-T_{c}^{\mathrm{MCT}}\right) \frac{\partial \gamma}{\partial p}\right]_{T} .
\end{gathered}
$$

In both expressions the diffusion coefficient extrapolates to zero, however, with one crucial difference. In the VFT expression, Eq. (4), this happens at $T_{0}^{\mathrm{VFT}}$, far below $T_{g}$, whereas in MCT diffusion vanishes at, $T_{c}^{\mathrm{MCT}}$, well above $T_{g}$. Because of the crossover to the glass the difference between the two expressions is normally not sufficient to distinguish between them with certainty. This is different for the pressure dependence where both expressions give singularities, below and above $T_{g}$, respectively.

There is only one measurement of $\hat{V}_{\text {act }}$ in the undercooled melt. Knorr et al. [14] find for $\mathrm{Ni}$ diffusion in the bulk metallic glass melt $\mathrm{Zr}_{46.75} \mathrm{Ti}_{8.25} \mathrm{Cu}_{7.5} \mathrm{Ni}_{10} \mathrm{Be}_{27.5}$ values between 0.35 and $0.64 \Omega$.

Whereas experiment so far only provides circumstantial evidence for the diffusion mechanism, molecular dynamics simulation can give both the quantities seen in experiment and show the underlying atomic processes. In a previous simulation on NiZr the activation volume was estimated as $0.36 \Omega$ from the change of barrier height [15]. The underlying process was a collective jump of a chain of atoms.

In the present paper we present a systematic study of the pressure dependence of diffusion as function of temperature. In order to relate closely to other work the simulations were done for the well-studied binary Lennard-Jones system (LJ), described by the interatomic interaction:

$$
V_{i j}(R)=4 \epsilon_{i j}\left[\left(\sigma_{i j} / R\right)^{12}-\left(\sigma_{i j} / R\right)^{6}+A_{i j} R+B_{i j}\right] .
$$

For the parameters $\epsilon_{i j}$ and $\sigma_{i j}$ we took the commonly used values of Kob and Andersen [16]: $\epsilon_{A A}=\epsilon=\sigma_{A A}=$ $\sigma=1, \epsilon_{B B}=0.5, \sigma_{B B}=0.88, \epsilon_{A B}=1.5$, and $\sigma_{A B}=$ 0.9. Different from these authors we use a larger cutoff radius $R_{c}=3 \sigma$. To avoid spurious cutoff effects we introduce, similar to the shifted force potential [17], the parameters $A_{i j}$ and $B_{i j}$ to ensure continuity of the potential and its first derivative at the cutoff. All masses were set to $m_{j}=1$. In the following, we will give all results in the reduced units of energy, $\epsilon_{A A}$, length, $\sigma_{A A}$, and atomic mass $m_{A}$.

We have used this potential previously to calculate the diffusional isotope effect parameter in the liquid [18] and the evolution of the dynamic heterogeneity both below and above the glass transition [19]. From these calculations we had well aged samples at different temperatures for zero external pressure.

The calculations were done at each temperature with constant volume, corresponding to zero pressure, a time step $\Delta t=0.005$ and periodic boundary conditions. The pressure was monitored. A heat bath was simulated by comparing the temperature, averaged over 20 time steps, with the nominal temperature. At each time step $1 \%$ of the temperature difference was adjusted by random additions to the particle velocities. This procedure assured that existing correlations between the motion of atoms were only minimally affected. At each temperature we had 8 independent samples, each of 5488 atoms in a ratio $4: 1$ of $A$ and $B$ atoms. The diffusion coefficients were calculated from the asymptotic slope of the atomic mean square displacements

$$
D(T)=\frac{1}{6 t} \lim _{t \rightarrow \infty}\left\langle\left(\mathbf{R}^{\ell}\left(t+t^{\prime}\right)-\mathbf{R}^{\ell}\left(t^{\prime}\right)\right\rangle_{\ell, t^{\prime}},\right.
$$

where $\langle\ldots\rangle_{\ell, t^{\prime}}$ indicates averaging over all atoms of the particular species and over starting times.

Figure 1 shows the densities and diffusion coefficients for the zero pressure configurations. From the change in slope of the volume expansion we estimate the glass transition temperature as $T_{g} \approx 0.35 \epsilon / \mathrm{k}$. The diffusion coefficient can be fitted very well both by the VFT and MCT expressions. For both fits we assume that the respective transition temperatures are the same for both species.

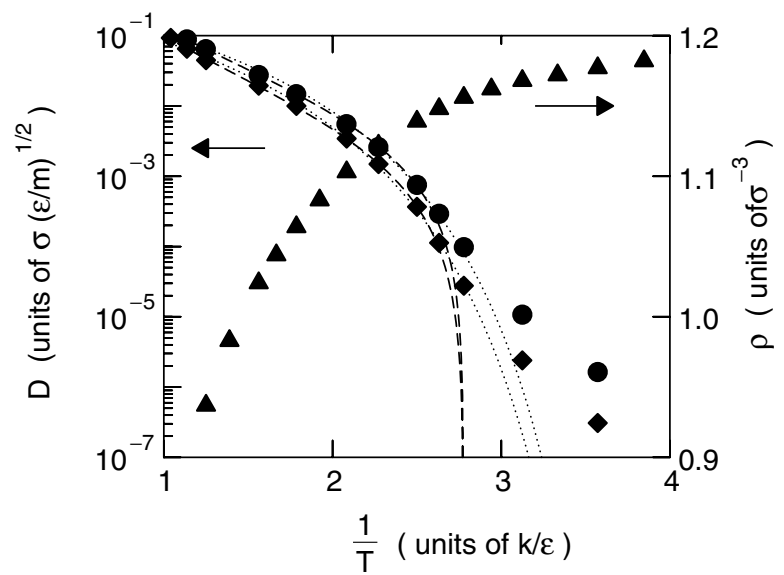

FIG. 1. Diffusion coefficients [majority $A$ atoms (diamonds) and minority $B$ atoms (circles)] and density (triangles) at zero pressure against inverse temperature (all in reduced units). The dashed and dotted lines show the fits with the MCT and VFT expressions, respectively. 
For the VFT expression, Eq. (4), we find the parameters $T_{0}^{\mathrm{VFT}}=0.244 \epsilon / k$ for both species and $E^{\mathrm{VFT}}=1.092$ and $0.986 \epsilon$ for $A$ and $B$ atoms, respectively. The values for the MCT expression, Eq. (6), are $T_{c}=0.36 \epsilon / k$ for both species and $\gamma=1.87$ and $\gamma=2.02$ for $A$ and $B$, respectively. The two temperatures, $T_{g}$ and $T_{c}$ are very close to each other, but are much lower than the value $T_{c}=0.435 \epsilon / k$, reported for simulations at constant density $\rho=1.2$ [20]. This reflects the strong dependence of the glass transition on density or pressure. We find for zero pressure a density of $\rho=1.15$ at $T_{g}$.

To calculate the pressure dependence we did additional runs with higher and lower densities, respectively. At each temperature the zero pressure samples were taken as starting configurations which were then compressed (expanded) and subsequently aged. The change of density was $2 \%$ at the highest and $0.5 \%$ at the lowest temperature. The resulting pressure change after aging was kept this way within the approximate limits of $\pm 0.2 \epsilon / \sigma^{3}$. The aging times are given in Table I.

After calculating the diffusion coefficients the activation volume was evaluated from Eq. (3). Figure 2 shows the resulting values together with the fit with the VFT expression, Eq. (5). One can clearly distinguish four temperature regions. At the highest temperature $\hat{V}_{\text {act }} \approx 0.6 \Omega$, nearly identical for both components. Upon cooling, it drops to a plateau value $\hat{V}_{\text {act }} \approx 0.3 \Omega$. The larger component has, as one would expect, a slightly higher activation volume. Below $T=0.5$ the activation volume rises sharply for both components and reaches a maximum of order $\Omega$ around $T=0.4$, near $T_{g}$ and $T_{c}^{\mathrm{MCT}}$. In the glassy state it drops again to a value below $0.3 \Omega$.

The drop of the activation volume in the liquid correlates with the drop of the isotope effect parameter reported for the same system [18]. The onset of the increase to the maximum near $T_{g}$ coincides with the onset of a pronounced curvature in the Arrhenius plot of $D(T)$, Fig. 1 . At this temperature and below the isotope effect parameter indicates collective motion [18]. From our previous work [21] and from the work on the LJ system under high pressure [22] we expect a predominance of chain motion with increasing chain lengths upon cooling. At these temperatures diffusion exhibits a pronounced dynamic heterogeneity over times of several $100\left(m \sigma^{2} / \epsilon\right)^{1 / 2}$ [19] which correlates with the time scale of the transition from $\beta$ to $\alpha$ relaxation [16].

Our values for the activation volume in the glass are similar to the estimate of Teichler [15] for the NiZr. Less well aged samples have somewhat higher $\hat{V}_{\text {act }}$. Previous

TABLE I. Aging times ( $t_{0}$ aging under zero pressure, $t_{p}$ subsequent aging after compression/dilatation).

\begin{tabular}{crrrr}
\hline \hline$T$ & $\geq 0.4$ & 0.36 & 0.32 & 0.28 \\
\hline$t_{0}\left(10^{4}\right.$ time steps $)$ & 6 & 67 & 126 & 300 \\
$t_{p}\left(10^{4}\right.$ time steps $)$ & 20 & 130 & 200 & 480 \\
\hline \hline
\end{tabular}

simulations of metallic glasses show collective jumps of chains of atoms $[15,23,24]$, an indication of an inherent collective diffusion mechanism.

The experimental values for $\mathrm{Ni}$ diffusion in the bulk glass $\mathrm{Zr}_{46.75} \mathrm{Ti}_{8.25} \mathrm{Cu}_{7.5} \mathrm{Ni}_{10} \mathrm{Be}_{27.5}$ [14] show an increase of the activation volume below $T=704 \mathrm{~K}$. When we plot (open circles in Fig. 2) the experimental values in LJ units by scaling with the temperature where the break in the diffusion coefficient occurs, i.e., without a free parameter, they closely follow the LJ result. In particular, they show the strong increase of $\hat{V}_{\text {act }}$ upon cooling towards $T_{c}^{\mathrm{MCT}}$. This apparent agreement poses, however, some questions. The two temperatures $T_{g}$ and $T_{c}$ nearly coincide in the LJ system, under the present quench and aging times. There is experimental evidence that in $\mathrm{Zr}_{46.75} \mathrm{Ti}_{8.25} \mathrm{Cu}_{7.5^{-}}$ $\mathrm{Ni}_{10} \mathrm{Be}_{27.5}$ the two temperatures differ strongly: $T_{g}=$ $592 \mathrm{~K}$ and $T_{c} \approx 875 \mathrm{~K}$ [25]. According to these numbers the increase of the activation volume would occur at $T_{g}$ and not at $T_{c}$ and, therefore, would not be the effect predicted by MCT. The experimental evidence is not sufficient to clear this point. There is a large experimental uncertainty on the measured values of the activation volume, the apparent increase might be an artifact of the fitting. Also the MCT critical temperature might be overestimated. Clearly better experimental values are needed.

As we have seen above, both VFT and MCT expressions predict the onset of a divergence in $\hat{V}_{\text {act }}(T)$. The dotted line in Fig. 2 shows a fit with the VFT expression, Eq. (5). We assume that the VFT temperatures are the same for both components, independent of pressure. The fit works relatively well. However, the resulting parameters are somewhat odd. We find $\partial T^{\mathrm{VFT}} / \partial p=0.3$ and $\partial E^{\mathrm{VFT}} / \partial p=$ 0 for both components and $\partial \ln D_{0}^{\mathrm{VFT}} / \partial p=-0.329$ and -0.2561 for the the $A$ and $B$ components, respectively.

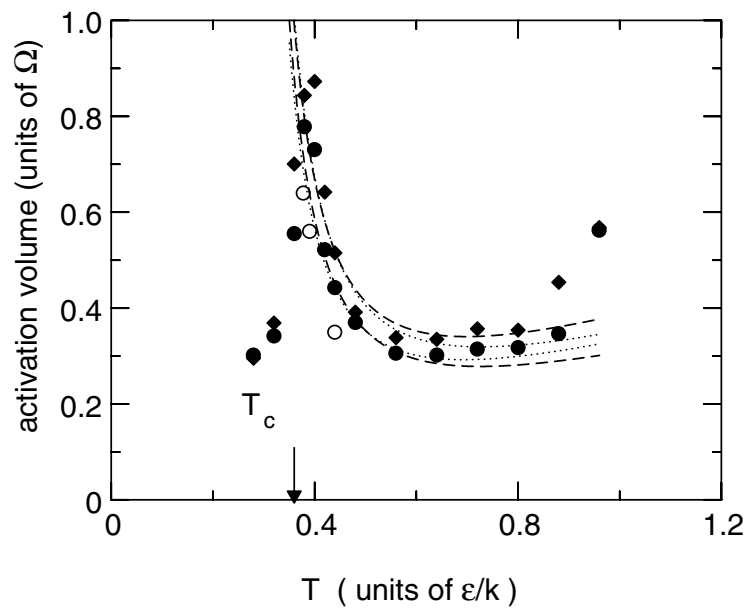

FIG. 2. Calculated activation volume [majority $A$ atoms (diamonds) and minority $B$ atoms (circles)] versus temperature. The open circles represent the experimental data on Ni diffusion in a bulk glass melt, scaled to LJ units. The dashed and dotted lines are fits with the VFT expression, with one or two VFT temperatures, respectively. 


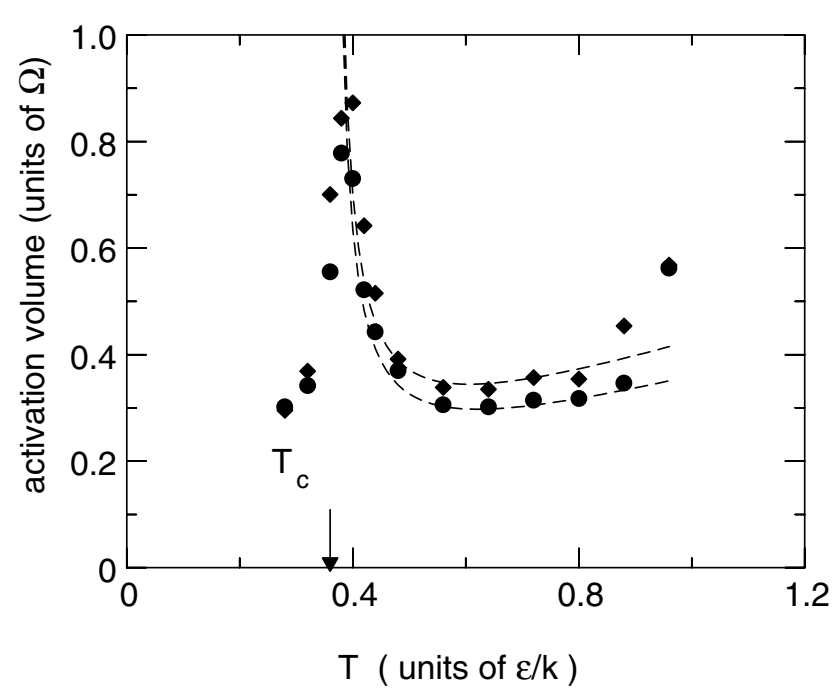

FIG. 3. Calculated activation volume [majority $A$ atoms (diamonds) and minority $B$ atoms (circles)] versus temperature. The dashed line is a fit with the MCT expression using a common critical temperature for both components.

This means, according to the fit there is very little change of the "activation energy" and a strong change of the prefactor. Allowing for different values of $\partial T^{\mathrm{VFT}} / \partial p$ the fit is somewhat improved. Its main shortcoming, the too slow rise, remains.

The alternative fit with the MCT expression, Eq. (7), is displayed in Fig. 3. As above, we assume a common value of $T_{c}^{\mathrm{MCT}}$ for both components and all pressures. We omitted in the fit the logarithmic term in Eq. (7), i.e., we neglected the weak dependence on a possible pressure variation of $\gamma$. We get an excellent fit (dashed line) with $\partial T_{c}^{\mathrm{MCT}} / \partial p=0.028$ for both components and $\partial \ln D_{0}^{\text {MCT }} / \partial p=-0.338$ and -0.278 for $A$ and $B$ atoms, respectively. The square deviation for this fit is an order of magnitude less than the one using the VFT expression. Contrary to the VFT case it reproduces the sharp uprise. We take this as an indication that the divergence of the activation volume would in deed be at $T_{c}^{\mathrm{MCT}}$ and not at $T^{\mathrm{VFT}}$. Of course the glass transition intervenes before the divergence is reached.

Kob and Andersen [16] report for their constant volume simulation a pressure of $p \approx 2.5$ at their $T_{c}^{\mathrm{MCT}}=0.435$. Extrapolating our value using the fitted pressure derivative we find $T_{c}^{\mathrm{MCT}}(p=2.5) \approx 0.43$ in excellent agreement with Kob and Andersen's value.

In conclusion, we find, both in the supercooled liquid and the glass, activation volumes of around 0.3 atomic volumes. This correlates with a high collectivity seen in the isotope effect. In the hot liquid, where diffusion is no longer by collective motion, the activation volume rises to about 0.6 atomic volumes. Cooling towards the critical temperature mode coupling theory predicts a $1 /\left(T-T_{c}\right)$ singularity, cut off by the glass transition. This is clearly observed in the simulation. This singular behavior can, e.g., be used to test whether there are one or several critical temperatures in a multicomponent system.

[1] H. Mehrer, Defect Diffus. Forum 129-130, 57 (1996).

[2] W. Frank, Defect Diffus. Forum 143-147, 695 (1997).

[3] Y. Loirat, J. L. Bocquet, and Y. Limoge, J. Non-Cryst. Solids 265, 252 (2000).

[4] F. Faupel, K. Rätzke, H. Ehmler, P. Klugkist, V. Zöllmer, C. Nagel, A. Rehmet, and A. Heesemann, Mater. Res. Soc. Symp. Proc. 664, L2.1.1 (2001).

[5] F. Faupel, W. Frank, M.-P. Macht, H. Mehrer, V. Naundorf, K. Rätzke, S. K. Sharma, H. R. Schober, and H. Teichler, Rev. Mod. Phys. (to be published).

[6] W. Götze and A. Sjölander, Rep. Prog. Phys. 55, 241 (1992).

[7] H. Mehrer, in Diffusion in Solid Metals and Alloys, edited by H. Mehrer, Landolt Börnstein, New Series, Group III, Vol. 26 (Springer, Berlin, 1990), p. 1.

[8] P. Klugkist, K. Rätzke, S. Rehders, P. Troche, and F. Faupel, Phys. Rev. Lett. 80, 3288 (1998).

[9] A. Heesemann, K. Rätzke, F. Faupel, J. Hoffmann, and K. Heinemann, Europhys. Lett. 29, 221 (1995).

[10] H. J. Höfler, R. S. Averback, G. Rummel, and H. Mehrer, Philos. Mag. Lett. 66, 301 (1992).

[11] A. Grandjean, P. Blanchard, and Y. Limoge, Phys. Rev. Lett. 78, 697 (1997).

[12] P. Klugkist, K. Rätzke, F. Faupel, P. Troche, P. Fielitz, and V. Naundorf, Philos. Mag. Lett. 79, 827 (1999).

[13] A. Heesemann, K. R. V. Zöllmer, and F. Faupel, Z. Metallkd. 91, 909 (2000).

[14] K. Knorr, M.-P. Macht, and H. Mehrer, in Materials Development and Processing - Bulk Amorphous Materials, Undercooling and Powder Metallurgy, edited by V. Woods, L. Schulz, and D. Herlach, EUROMAT99 (Wiley-VCH, Weinheim, 2000), Vol. 8, p. 1.

[15] H. Teichler, J. Non-Cryst. Solids (to be published).

[16] W. Kob and H. Andersen, Phys. Rev. E 51, 4626 (1995).

[17] J. J. Nicolas, K. E. Gubbins, W. B. Streett, and D. J. Tildesley, Mol. Phys. 37, 1429 (1979).

[18] H. R. Schober, Solid State Commun. 119, 73 (2001).

[19] D. Caprion, J. Matsui, and H. R. Schober, Phys. Rev. Lett. 85, 4293 (2000).

[20] W. Kob and H. Andersen, Phys. Rev. E 52, 4134 (1995).

[21] H. R. Schober, C. Gaukel, and C. Oligschleger, Defect Diffus. Forum 143-147, 723 (1997).

[22] C. Donati, J.F. Douglas, W. Kob, S. J. Plimpton, P. H. Poole, and S. C. Glotzer, Phys. Rev. Lett. 80, 2338 (1998).

[23] H. R. Schober, C. Oligschleger, and B. B. Laird, J. NonCryst. Solids 156, 965 (1993).

[24] C. Oligschleger and H. R. Schober, Phys. Rev. B 59, 811 (1999).

[25] A. Meyer, J. Wuttke, W. Petry, O. G. Randl, and H. Schober, Phys. Rev. Lett. 80, 4454 (1998). 